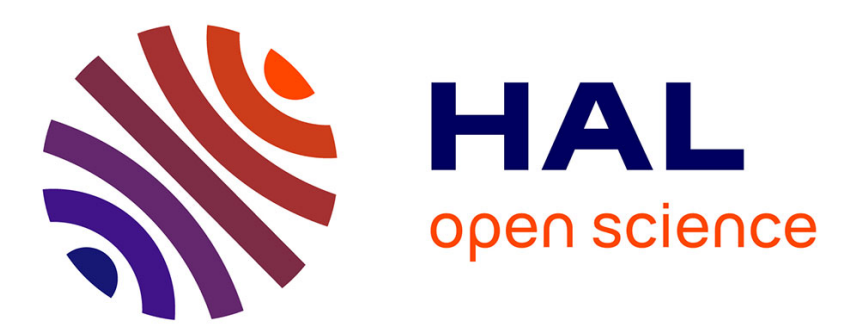

\title{
On the identification of the electron temperature profile from polarimetry Stokes vector measurements in Tokamak free-boundary equilibrium reconstruction
} Blaise Faugeras, Francesco Orsitto

\section{- To cite this version:}

Blaise Faugeras, Francesco Orsitto. On the identification of the electron temperature profile from polarimetry Stokes vector measurements in Tokamak free-boundary equilibrium reconstruction. Plasma Physics and Controlled Fusion, 2019, 61 (11), pp.115002. 10.1088/1361-6587/ab411a . hal-02395035

\section{HAL Id: hal-02395035 \\ https://hal.science/hal-02395035}

Submitted on 19 Dec 2019

HAL is a multi-disciplinary open access archive for the deposit and dissemination of scientific research documents, whether they are published or not. The documents may come from teaching and research institutions in France or abroad, or from public or private research centers.
L'archive ouverte pluridisciplinaire HAL, est destinée au dépôt et à la diffusion de documents scientifiques de niveau recherche, publiés ou non, émanant des établissements d'enseignement et de recherche français ou étrangers, des laboratoires publics ou privés. 


\title{
On the identification of the electron temperature profile from polarimetry Stokes vector measurements in Tokamak free-boundary equilibrium reconstruction
}

\author{
Blaise Faugeras ${ }^{1}$ and Francesco Orsitto ${ }^{2}$ \\ ${ }^{1}$ Laboratoire J.A. Dieudonné, Université Côte d'Azur, CNRS, Inria, Parc Valrose, \\ 06108 Nice Cedex 2, France \\ ${ }^{2}$ CREATE Consortium, Universita di Napoli Federico II Naples and ENEA \\ Depertment FSN Frascati, Italy \\ E-mail: blaise.faugeras@unice.fr
}

\begin{abstract}
This paper reports numerical investigations on the identification of the electron temperature profile $T_{e}$ from interferometry and polarimetry Stokes vector measurements with the equilibrium code NICE. This latter enables the consistent resolution of the inverse equilibrium reconstruction problem in the framework of nonlinear free-boundary equilibrium coupled to the Stokes model equation for polarimetry. We find that for ITER plasma with high $I_{p}, N_{e}$ and $T_{e}$ the identification from noisy measurements is possible.
\end{abstract}

Keywords: equilibirum reconstruction, polarimetry, Stokes model, electron temperature profile identification

\section{Introduction}

The high levels of plasma current, electron density and electron temperature foreseen in ITER plasma regimes impose that the Stokes model should be used for polarimetry $[1,2,3,4,5]$ instead of its approximation giving the Faraday rotation angle formula usually used in equilibrium reconstruction.

Equilibrium reconstruction using more elaborated polarimetry models than the Faraday angle only have already been proposed and tested in $[6,7,8]$ on simplified plasma models and in $[9,10]$ for the complete framework of non-linear free-boundary equilibrium coupled to the Stokes model equation in the code NICE (Newton direct and Inverse Computation for Equilibrium).

The goal of the present paper is to investigate numerically the possibility to identify the electron temperature profile $T_{e}$ from interferometry and polarimetry Stokes vector measurements. In [7] it has been shown on a simplified case (fixed boundary plasma 
equilibrium, no interferometry measurements, no noise on measurements) that it is possible to extract information on the $T_{e}$ profile from polarimetry measurements. Since these measurements are routinely available during a shot the perspective of using freeboundary equilibrium reconstruction tools to identify the $T_{e}$ profile is very interesting. For the present work the Stokes model already implemented in NICE was modified in order to take into account $T_{e}$ effects. The dependence of interferometric measurements on $T_{e}$ is also taken into account [2].

The outline of the paper is as follows. Next Section 2 is devoted to the formulation of the direct model. Except for the $T_{e}$ dependence of the Stokes model it is very close to Section 2 from our previous paper [10] but needed for the rest of the present paper. Section 3 deals with the inverse equilibrium reconstruction and $T_{e}$ identification problem. Eventually Section 4 starts with a brief description of the numerical methods implemented in NICE and then presents results for several numerical experiments.

\section{Direct model}

Free-boundary plasma equilibrium Assuming axial symmetry and introducing a cylindrical coordinate system $\left(\boldsymbol{e}_{r}, \boldsymbol{e}_{\phi}, \boldsymbol{e}_{z}\right)$ we consider the classical non-linear freeboundary Grad-Shafranov equilibrium model for the poloidal flux $\psi(r, z)$

$$
\begin{cases}-\Delta^{*} \psi=\left[\lambda\left(\frac{r}{r_{0}} \mathcal{A}\left(\psi_{\mathrm{N}}\right)+\frac{r_{0}}{r} \mathcal{B}\left(\psi_{\mathrm{N}}\right)\right)\right] 1_{\Omega_{\mathrm{p}}(\psi)}+\sum_{i} \frac{I_{i}}{S_{i}} 1_{\Omega_{c_{i}}} & \text { in } \Omega \\ \text { boundary conditions for } \psi & \text { on } \partial \Omega,\end{cases}
$$

Here $\Delta^{*}$ is defined by

$$
\Delta^{*} .:=\nabla \cdot\left(\frac{1}{\mu_{0} r} \nabla .\right) .
$$

$\nabla$ is the $2 \mathrm{D}$ operator in the $(r, z)$-plane and $\mu_{0}$ is the magnetic permeability of vacuum.

The computational domain $\Omega$ contains the limiter domain $\Omega_{\mathrm{L}}$ accessible to the plasma. It can also contain poloidal field coils $\Omega_{\mathrm{c}_{i}}$ with section $S_{i}$ and currents $I_{i}$.

The first term in the right hand side of Eq. (1) represents the toroidal component of the current density in the plasma,

$$
j_{\phi}=r p^{\prime}(\psi)+\frac{1}{r \mu_{0}} f f^{\prime}(\psi),
$$

expressed using the adimentionalized non linear functions $\mathcal{A}$ and $\mathcal{B}$, the major radius $r_{0}$ and a scaling factor $\lambda .1_{\Omega_{\mathrm{p}}(\psi)}$ is the indicator function of the unknown plasma domain. This domain is defined by its boundary which is the outermost closed $\psi$ iso-contour contained within the limiter domain $\Omega_{\mathrm{L}}$.

The normalized poloidal flux $\psi_{\mathrm{N}}(r, z)$ is

$$
\psi_{\mathrm{N}}(r, z)=\frac{\psi(r, z)-\psi_{\mathrm{a}}}{\psi_{\mathrm{b}}-\psi_{\mathrm{a}}},
$$

with $\psi_{\mathrm{a}}$ and $\psi_{\mathrm{b}}$ being the flux values at the magnetic axis and at the boundary of the plasma. 
Stokes model for polarimetry Polarimetry consists in measurements of the change of state of polarization of an electromagnetic radiation propagating across the magnetized plasma along several lines of sight (or chords) distributed on the poloidal section of the tokamak. One method of describing the state of polarization is to introduce a Stokes vector $\mathbf{s}=\left(s_{1}, s_{2}, s_{3}\right)$. The evolution of the polarization when the laser beam crosses the plasma is then given by the following Stokes equation on each line of sight:

$$
\left\{\begin{array}{l}
\frac{d \mathbf{s}}{d Z}=\mathbf{G} \mathbf{s}, \quad \text { on }\left(Z_{0}, Z_{1}\right], \\
\mathbf{s}\left(Z_{0}\right)=\mathbf{s}_{0} .
\end{array}\right.
$$

We refer to [1] for details on this modelization. Here we have introduced a coordinate system $\left(\boldsymbol{e}_{X}, \boldsymbol{e}_{Y}, \boldsymbol{e}_{Z}\right)$ attached to a line of sight $L . Z$ is the coordinate tangent to the chord, $X$ represents the toroidal direction and $Y$ the direction perpendicular to $Z$ in the poloidal plane. In this coordinate system the components of the magnetic field are denoted by $\left(B_{X}, B_{Y}, B_{Z}\right)$.

The initial polarization is given by $\mathbf{s}_{0}$ at $Z_{0}$. $Z_{1}$ corresponds to the location of the output measurement sensor. The $3 \times 3$ matrix $\boldsymbol{G}$ is such that $\boldsymbol{G} \mathbf{s}=\boldsymbol{\Omega} \times \mathbf{s}$ where vector $\boldsymbol{\Omega}=\left(\Omega_{1}, \Omega_{2}, \Omega_{3}\right)$ has components [7]:

$$
\Omega_{1}^{c}:=c_{1} N_{e}\left(B_{X}^{2}-B_{Y}^{2}\right), \quad \Omega_{2}^{c}:=2 c_{1} N_{e} B_{X} B_{Y}, \quad \Omega_{3}^{c}:=c_{3} N_{e} B_{Z},
$$

in the low electron temperature approximation and components

$$
\Omega_{1}=\left(1+\frac{9 T_{e}}{2 m_{e} c^{2}}\right) \Omega_{1}^{c}, \quad \Omega_{2}=\left(1+\frac{9 T_{e}}{2 m_{e} c^{2}}\right) \Omega_{2}^{c}, \quad \Omega_{3}=\left(1-2 \frac{T_{e}}{m_{e} c^{2}}\right) \Omega_{3}^{c},
$$

in the high electron temperature approximation $(T e \gtrsim 5[\mathrm{keV}])$.

Here the electron density and temperature in the plasma, $N_{e}=N_{e}\left(\psi_{\mathrm{N}}\right)$ and $T_{e}=T_{e}\left(\psi_{\mathrm{N}}\right)$, are assumed to be constant on flux surfaces. The components of the magnetic field can be written as

$$
B_{Z}=-\frac{1}{r} \nabla \psi \cdot \boldsymbol{e}_{Y}, \quad B_{Y}=\frac{1}{r} \nabla \psi \cdot \boldsymbol{e}_{Z}, \quad B_{X}=\frac{f}{r}
$$

where the diamagnetic function $f$ is related to function $\mathcal{B}$ from Eq. (1) through the relation $f f^{\prime}=\lambda \mu_{0} r_{0} \mathcal{B}$. In order to keep notations consistant let us also introduce normalizing constants, $\lambda_{N_{e}}$ and $\lambda_{T_{e}}$, and functions, $\mathcal{C}$ and $\mathcal{D}$, such that $N_{e}\left(\psi_{\mathrm{N}}\right)=$ $\lambda_{N_{e}} \mathcal{C}\left(\psi_{\mathrm{N}}\right)$ and $T_{e}\left(\psi_{\mathrm{N}}\right)=\lambda_{T_{e}} \mathcal{D}\left(\psi_{\mathrm{N}}\right)$. The dependence of $\boldsymbol{G}$ on $\psi$, on the electron density function $\mathcal{C}$, on the electron temperature function $\mathcal{D}$ and on function $\mathcal{B}$ is denoted by $\boldsymbol{G}(\psi, \mathcal{B}, \mathcal{C}, \mathcal{D})$. Constants $c_{1}$ and $c_{3}$ depend on the wavelength of the beam radiation.

In order to use polarimetry measurements with Stokes modelization for the equilibrium reconstruction problem one has to supplement equation (1) with, for each interfero-polarimetry line of sight, a system of linear ordinary differential equations (2) for the Stokes vector.

\section{Inverse identification problem}

Equilibirum reconstruction together with the identification of functions $\mathcal{A}, \mathcal{B}$ and $\mathcal{C}$ from magnetic measurements and interfero-polarimetry measurements is a well known and 
studied problem. On the other hand the inclusion of electron temperature in the Stokes model and the possibility to identify it has been much less investigated.

The identification problem is formulated as a constrained optimization problem for the cost function

$$
J(\psi,\{\mathbf{s}\}, \mathcal{A}, \mathcal{B}, \mathcal{C}, \mathcal{D}):=J_{\text {mag }}(\psi)+J_{\text {interf }}(\psi, \mathcal{C}, \mathcal{D})+J_{S}(\{\mathbf{s}\})+R(\mathcal{A}, \mathcal{B}, \mathcal{C}, \mathcal{D}),
$$

with $\{\mathbf{s}\}$ denoting the vector $\left(\mathbf{s}^{1}, \ldots, \mathbf{s}^{N_{L}}\right)$ of Stokes vectors for all $N_{L}$ chords.

$J_{\text {mag }}(\psi)$ is the classical least square misfit term on magnetic measurements which constitutes the basic set of experimental measurements used in equilibrium reconstruction for the identification of functions $\mathcal{A}$ and $\mathcal{B}$.

$J_{\text {interf }}(\psi, \mathcal{C}, \mathcal{D})$ is the least square misfit term on line integrated interferometric measurements for each chord. It depends on $N_{e}\left(=\lambda_{N_{e}} \mathcal{C}\right)$ and on $T_{e}\left(=\lambda_{T_{e}} \mathcal{D}\right)$ since, refering to [2], at high electron temperature interferometric measurements along a chord $L$ are given by

$$
\int_{L} N_{e}\left(\psi_{\mathrm{N}}\right)\left(1-\frac{3}{2} \frac{T_{e}\left(\psi_{\mathrm{N}}\right)}{m_{e} c^{2}}\right) d Z
$$

Polarimetric Stokes measurements are given by the full Stokes vector at the $Z_{1}^{i}$ coordinate on each chord, $\mathbf{s}_{o b s}^{i} \approx \mathbf{s}\left(Z_{1}^{i}\right)$ and $J_{S}(\{\mathbf{s}\})$ is the least square misfit term on these measurements.

Finally $R(\mathcal{A}, \mathcal{B}, \mathcal{C}, \mathcal{D})$ is a regularization term ensuring the smoothness of the reconstructed profiles [9].

The equilibrium reconstruction problem using Stokes model with electron temperature for polarimetry is formulated as:

Reconstruction problem. Find $(\psi,\{\mathbf{s}\}, \mathcal{A}, \mathcal{B}, \mathcal{C}, \mathcal{D})$

minimizing $J(\psi,\{\mathbf{s}\}, \mathcal{A}, \mathcal{B}, \mathcal{C}, \mathcal{D})$ from $E q$. (6)

under the constraint of the model equations (7) and (8) below:

$$
\begin{cases}-\Delta^{*} \psi=\left[\lambda\left(\frac{r}{r_{0}} \mathcal{A}\left(\psi_{\mathrm{N}}\right)+\frac{r_{0}}{r} \mathcal{B}\left(\psi_{\mathrm{N}}\right)\right)\right] 1_{\Omega_{\mathrm{p}}(\psi)}+\sum_{i} \frac{I_{i}}{S_{i}} 1_{\Omega_{c_{i}}} & \text { in } \Omega \\ \text { boundary conditions for } \psi & \text { on } \partial \Omega,\end{cases}
$$

and for all lines of sight $L^{i}, i=1, \ldots N_{L}$ :

$$
\left\{\begin{array}{l}
\frac{d \mathbf{s}^{i}}{d Z^{i}}=\boldsymbol{G}(\psi, \mathcal{B}, \mathcal{C}, \mathcal{D}) \mathbf{s}^{i}, \quad \text { on }\left(Z_{0}^{i}, Z_{1}^{i}\right] \\
\mathbf{s}^{i}\left(Z_{0}^{i}\right)=\mathbf{s}_{0}^{i}
\end{array}\right.
$$

The unknown functions $\mathcal{A}, \mathcal{B}, \mathcal{C}$ and $\mathcal{D}$ are supposed to belong to a set $\mathcal{U}$ of regular functions defined on $[0,1]$.

\section{Numerical results}

Numerical solution method The numerical methods used in this work are the ones presented in $[9,10,11]$ implemented in the code NICE and extended to take into account 
electron temperature in the polarimetry Stokes model as well as in the interferometry model.

Basically we use finite elements $[12,13,14]$ for the discretization of equilibrium equation (7) and the Crank-Nicolson scheme for equation (8). An iterative sequential quadratric programming method (SQP) $[15,16]$ is implemented to solve the optimization problem for the non-quadratic cost function under the constraint of the non-linear model. Optimal $\mathcal{A}, \mathcal{B}, \mathcal{C}$ and $\mathcal{D}$ functions are found at convergence of the iterations and error bars on theses reconstructed functions can be computed from the Hessian matrix of the reduced cost function.

ITER Synthetic magnetic, interferometry and polarimetry measurements are computed using input reference profiles $\mathcal{A}, \mathcal{B}, \mathcal{C}$ and $\mathcal{D}$ to solve the direct model (7) and (8). The setting is the same as in [9]: plasma current $I_{p}=15[\mathrm{MA}]$, central density $N_{e}(0)=10^{20}\left[\mathrm{~m}^{-3}\right], 15$ interfero-polarimetry chords (Figure 3 from [9] shows the computed reference plasma). Concerning electron temperature it is given as $T_{e}\left(\psi_{\mathrm{N}}\right)=\lambda_{T_{e}} \mathcal{D}\left(\psi_{\mathrm{N}}\right)$ with $\mathcal{D}\left(\psi_{\mathrm{N}}\right)=\left(1-\psi_{\mathrm{N}}^{3}\right)$ and $\lambda_{T_{e}}=5,10,20$ or $30[\mathrm{keV}]$.

Figures 1, 2, 3 and 4 show the simulated intereferometry and Stokes vector measurements without noise and for the different $\lambda_{T_{e}}$ values considered. The effect of the inclusion of $T_{e}$ in the modelization is visible. Increasing $T_{e}$ clearly decreases interferometry values as expected. On the contrary no general trend on Stokes vector components can be observed. Some components are highly perturbated by increasing $T_{e}$ whereas other or less perturbated.

In a second step the equilibrium reconstruction algorithm is run using these input synthetic measurement to which noise can be added. The inclusion of $T_{e}$ in the modelization is a perturbation of interfero-polarimetry measurements only and in any case the algorithm identifies correctly the 3 other profiles $\mathcal{A}, \mathcal{B}$ and $\mathcal{C}$. As an example the reconstructed electron density profiles are shown on figures $5,6,7$ and 8 for different noise levels on interfero-polarimetry and different values of $\lambda_{T_{e}}$. More importantly the objective of this study is to see how well the $T_{e}$ profile can be recovered.

Figure 9 shows the reference and computed profiles for the $4 \lambda_{T_{e}}$ values and without additional noise on the measurements. The reconstruction is excellent and this can be considered as a sanity check for the algorithm. It can be seen from the second column of Table 1 that the errors on the identified $T_{e}$ profile are very low, the highest value being of $2.5 \%$ for the $\lambda_{T_{e}}=5$ case. This is the most difficult case since the perturbation induced by electron temperature on the Stokes vector is the smallest.

Figures 10, 11 and 12 show the mean (over 10 reconstructions) $T_{e}$ and error bar profiles for experiments with additional 1\%,5\% and $10 \%$ noise on interferometry and polarimetry measurements and $1 \%$ noise on magnetics. Table 1 also shows the relative errors for these cases. In the first case $\left(1 \%\right.$ noise) the identification of the $T_{e}$ profile is excellent. For the second case ( $5 \%$ noise) and third case (10\% noise) the results are degraded and particularly in the most difficult situation of the lowest $\lambda_{T_{e}}$ value.

The effect of noise on measurements can also be understood from Figure 13 where 
for each of the 3 components of the Stokes vector for each of the 15 chords (hence 45 values on the $\mathrm{X}$-axis) are plotted: $0.01,0.05$ and 0.1 times the result of the model without $T_{e}$ (denoted $|S 0|$ ) as well as the difference between the model with $T_{e}$ for $\lambda_{T_{e}}=5,10,20$ and 30 , and the model without $T_{e}$. The horizontal line shows the mean value over the 45 components. The mean values $|S 5-S 0|$ and $|S 10-S 0|$ are lower than $0.1 \times|S 0|$. This shows that $10 \%$ noise on Stokes vector measurements can hide the influence of $T_{e}$ on the model. On the contrary most dots are above the mean $0.01 \times|S 0|$ value showing that a $1 \%$ noise on measurements should not forbid the correct identification of the $T_{e}$ profile.

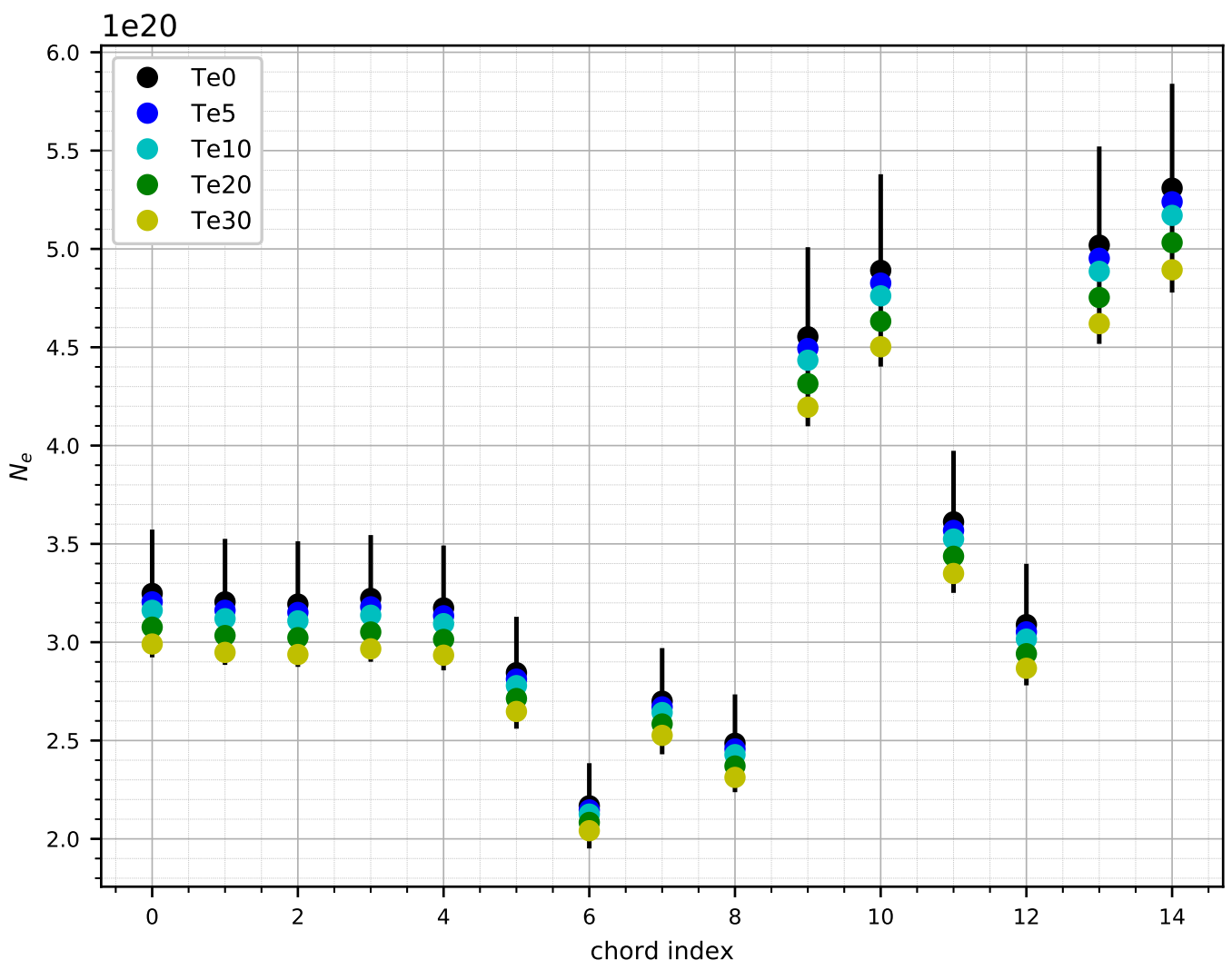

Figure 1. Effect of $T_{e}$ on simulated line integrated electron density measurements. For each chord (index 0 to 14 on X-axis) the $N_{e}$ values using the model with $\lambda_{T_{e}}=0$, $5,10,20$ and 30 are plotted. The vertical black segments shows $10 \%$ variation around the $\lambda_{T_{e}}=0$ value. 


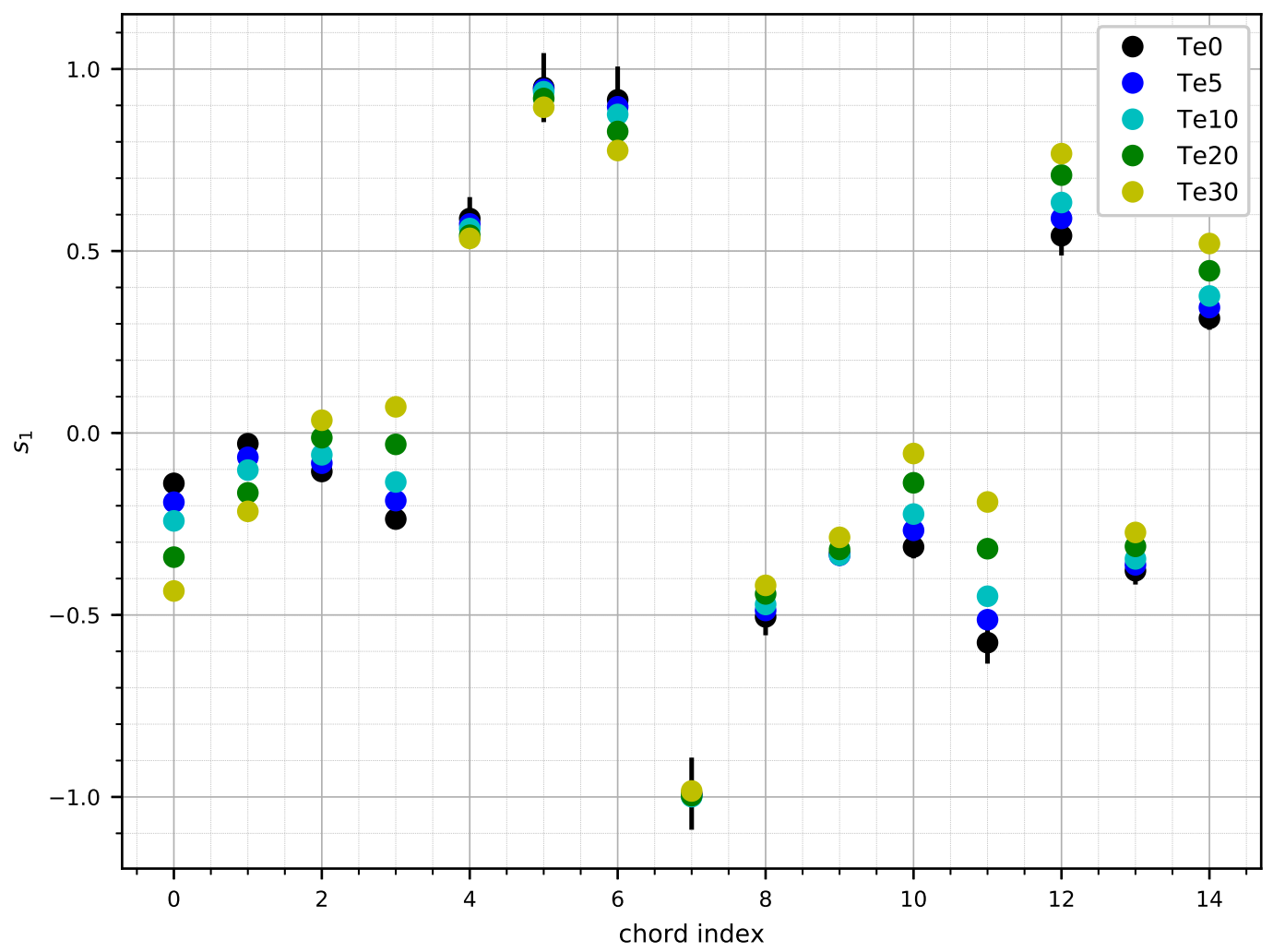

Figure 2. Effect of $T_{e}$ on simulated first component $s_{1}$ of the Stokes vector measurements. For each chord (index 0 to 14 on X-axis) the $s_{1}$ values using the model with $\lambda_{T_{e}}=0,5,10,20$ and 30 are plotted. The vertical black segments shows $10 \%$ variation around the $\lambda_{T_{e}}=0$ value.

\begin{tabular}{|c|c|c|c|c|}
\hline$\lambda_{T_{e}}$ & no noise & $1 \%$ & $5 \%$ & $10 \%$ \\
\hline 5 & 0.025 & 0.053 & 0.198 & 0.310 \\
10 & 0.015 & 0.018 & 0.092 & 0.178 \\
20 & 0.008 & 0.010 & 0.032 & 0.089 \\
30 & 0.006 & 0.008 & 0.026 & 0.015 \\
\hline
\end{tabular}

Table 1. Relative error on the identified $T_{e}$ profile, $\frac{\left\|T_{e}-T_{e}^{r e f}\right\|}{\left\|T_{e}^{r e f}\right\|}$, for varying $\lambda_{T_{e}}$ and experiments with $0,1,5$ and $10 \%$ additional noise on interferometry and polarimetry measurements. 


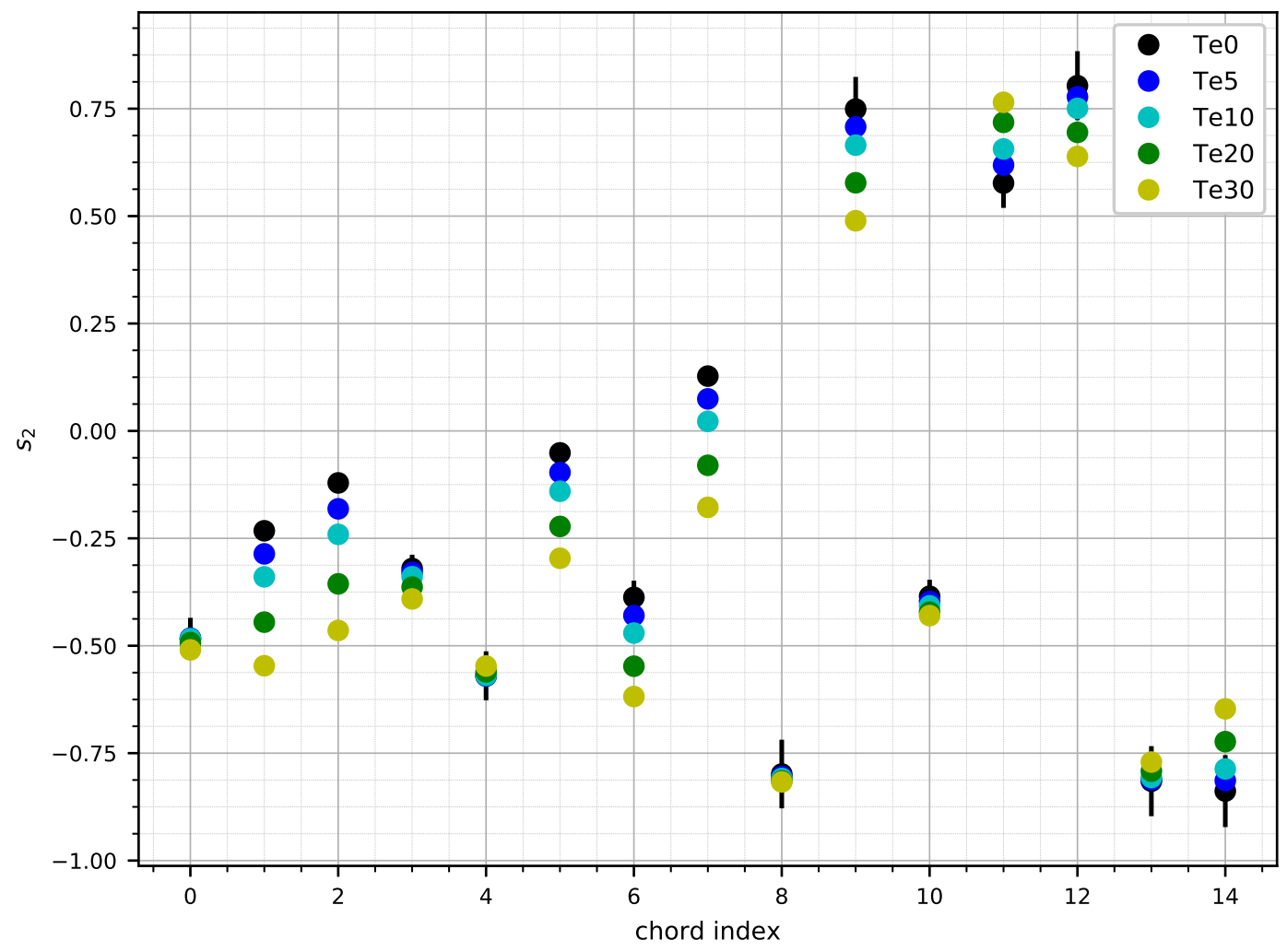

Figure 3. Effect of $T_{e}$ on simulated second component $s_{2}$ of the Stokes vector measurements. For each chord (index 0 to 14 on X-axis) the $s_{2}$ values using the model with $\lambda_{T_{e}}=0,5,10,20$ and 30 are plotted. The vertical black segments shows $10 \%$ variation around the $\lambda_{T_{e}}=0$ value. 


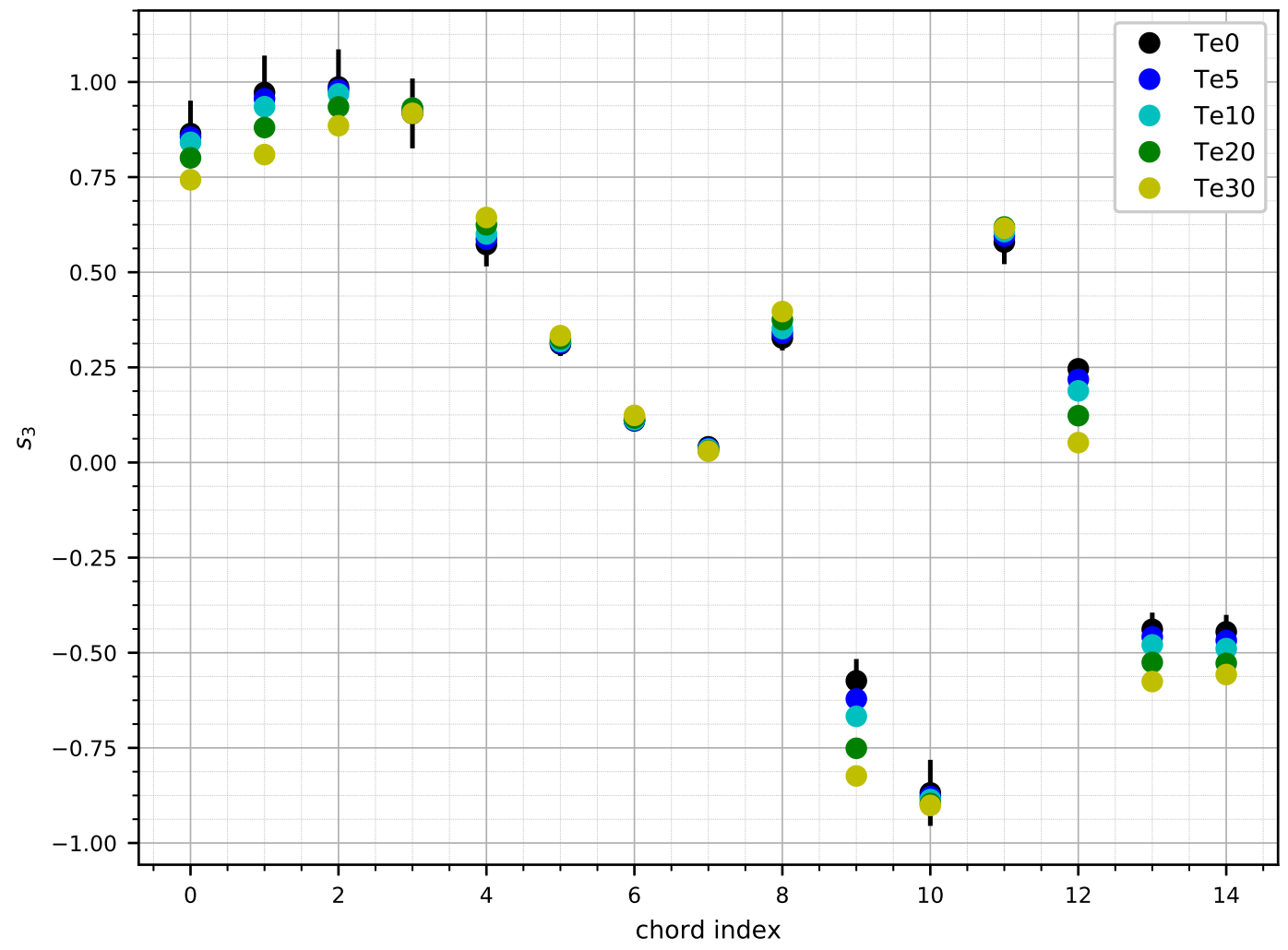

Figure 4. Effect of $T_{e}$ on simulated third component $s_{3}$ of the Stokes vector measurements. For each chord (index 0 to 14 on $\mathrm{X}$-axis) the $s_{3}$ values using the model with $\lambda_{T_{e}}=0,5,10,20$ and 30 are plotted. The vertical black segments shows $10 \%$ variation around the $\lambda_{T_{e}}=0$ value. 

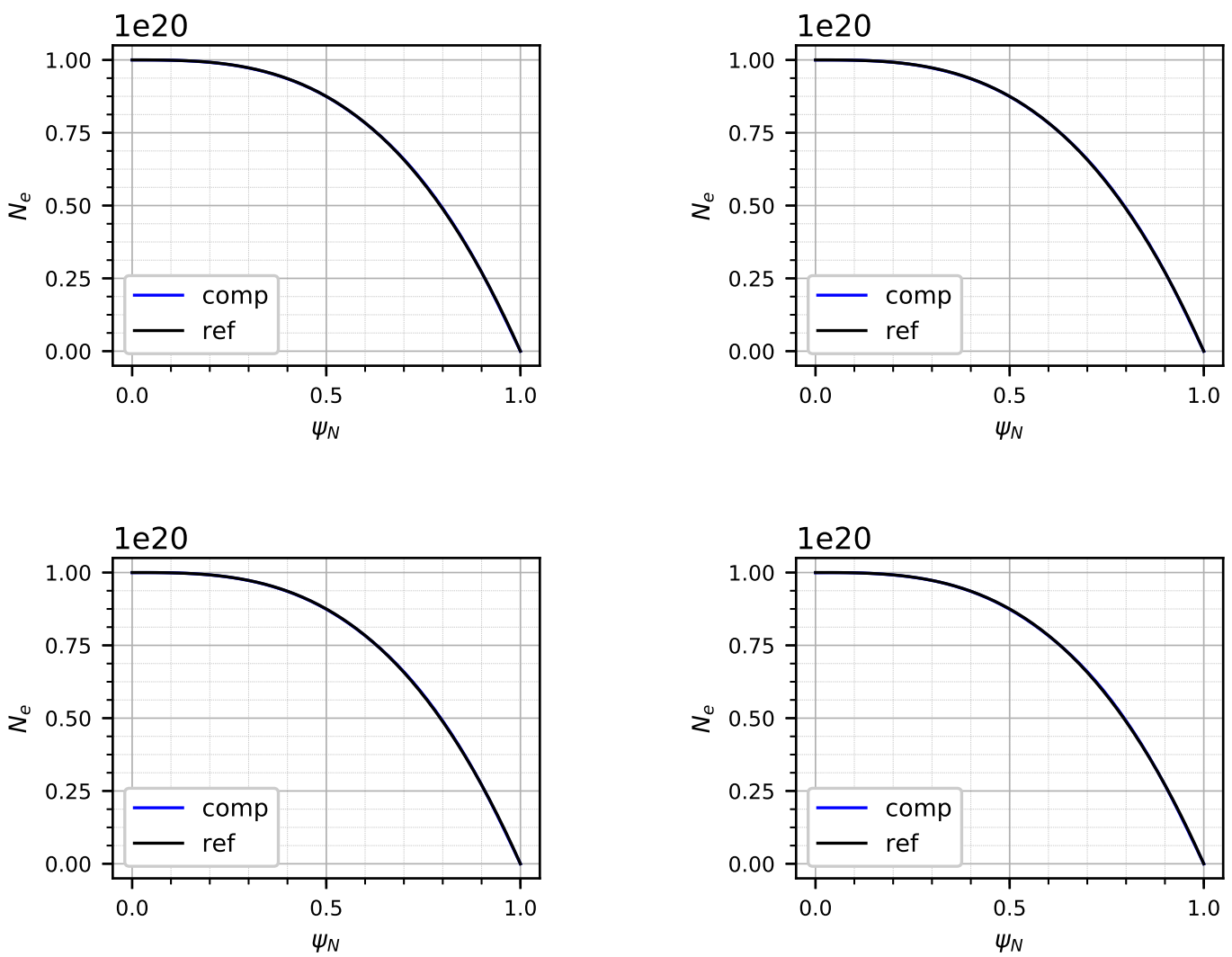

Figure 5. Identified and reference $N_{e}$ profile for $\lambda_{T_{e}}=5,10,20$ and 30 (top left to bottom right). Experiments without additional noise on interferometry and polarimetry measurements. 

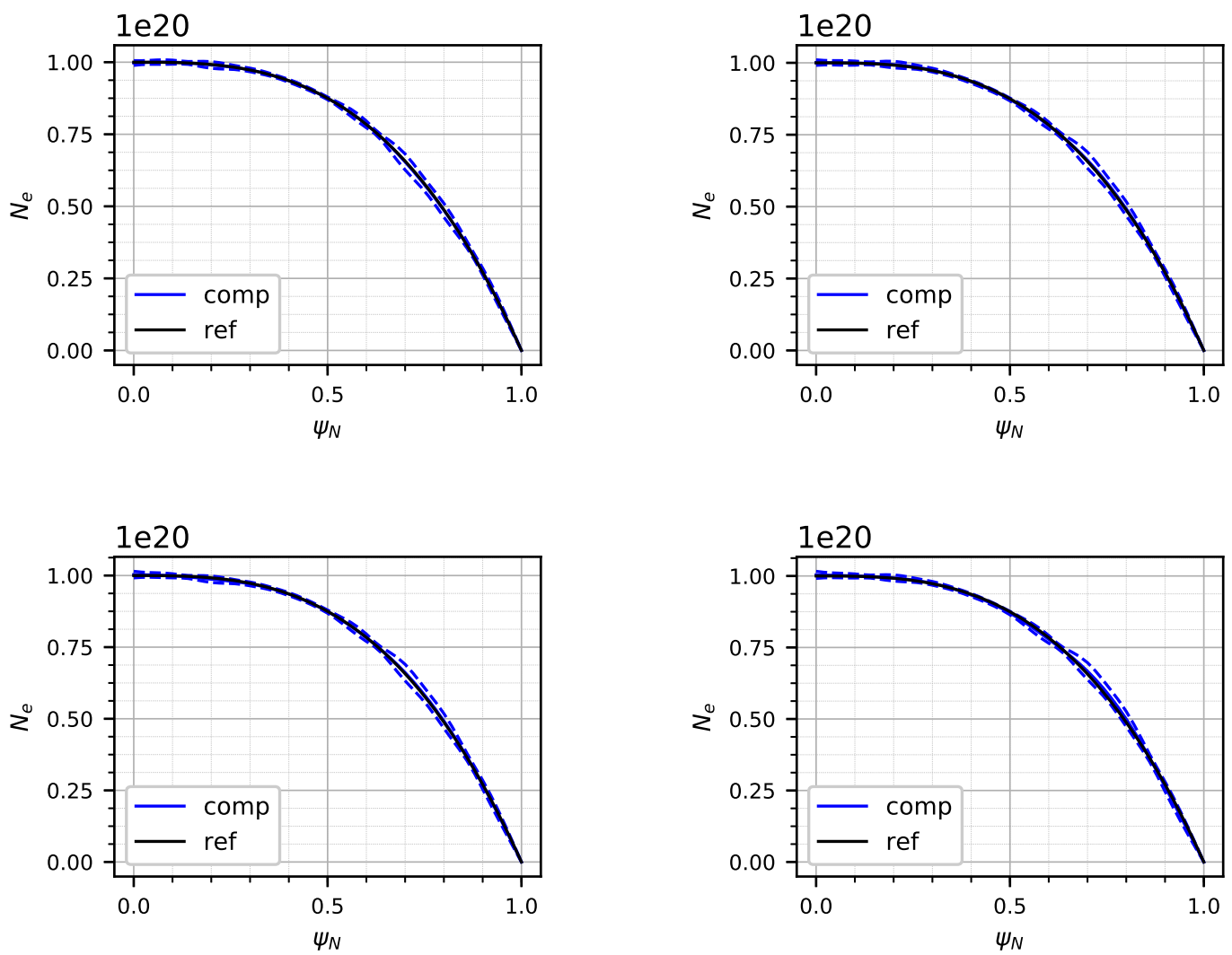

Figure 6. Mean (over 10 reconstructions) identified $N_{e}$ profile and error bar profiles, reference $N_{e}^{r e f}$ profile for $\lambda_{T_{e}}=5,10,20$ and 30 (top left to bottom right). Experiments with $1 \%$ additional noise on interferometry and polarimetry measurements. 

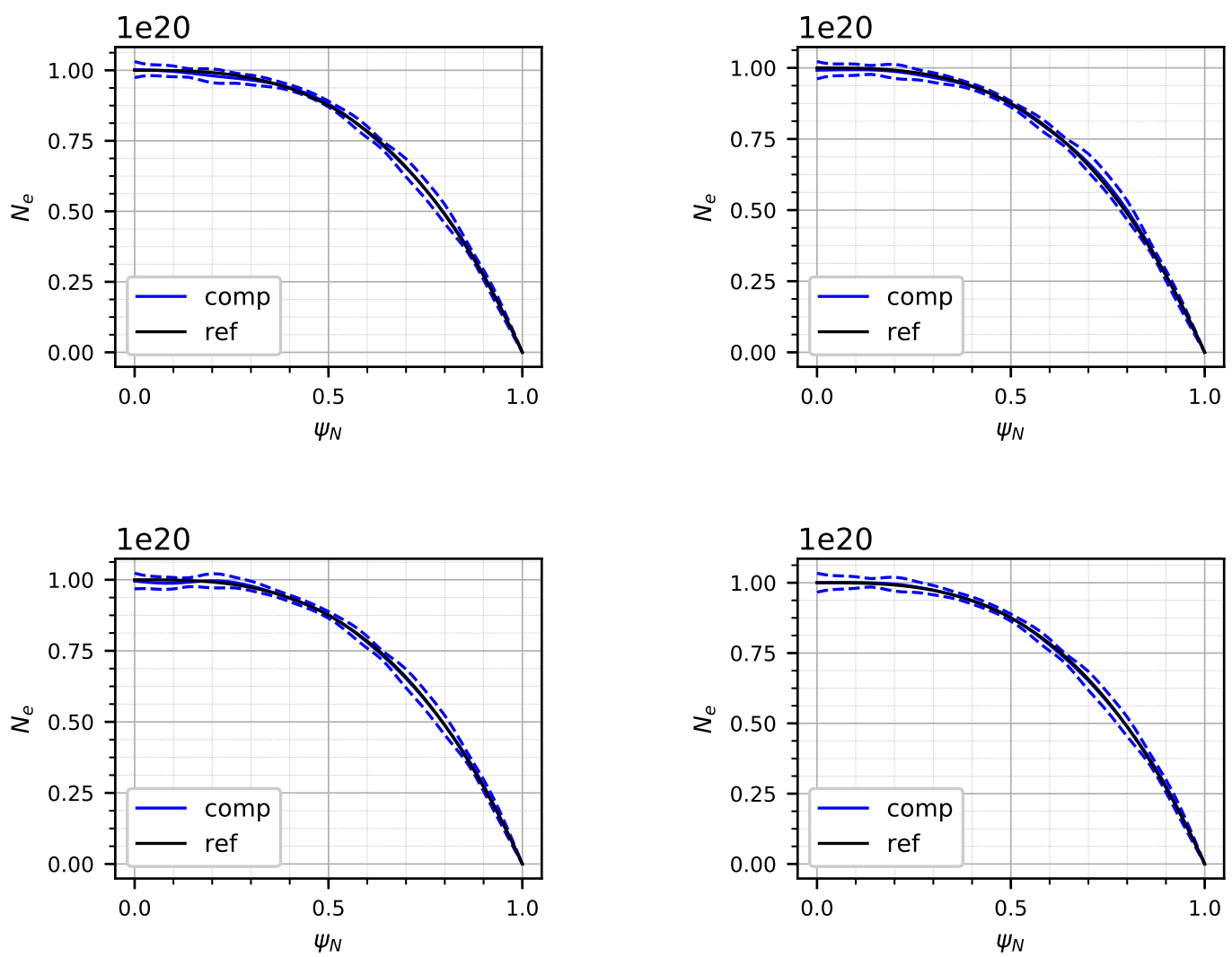

Figure 7. Mean (over 10 reconstructions) identified $N_{e}$ profile and error bar profiles, reference $N_{e}^{r e f}$ profile for $\lambda_{T_{e}}=5,10,20$ and 30 (top left to bottom right). Experiments with $5 \%$ additional noise on interferometry and polarimetry measurements. 

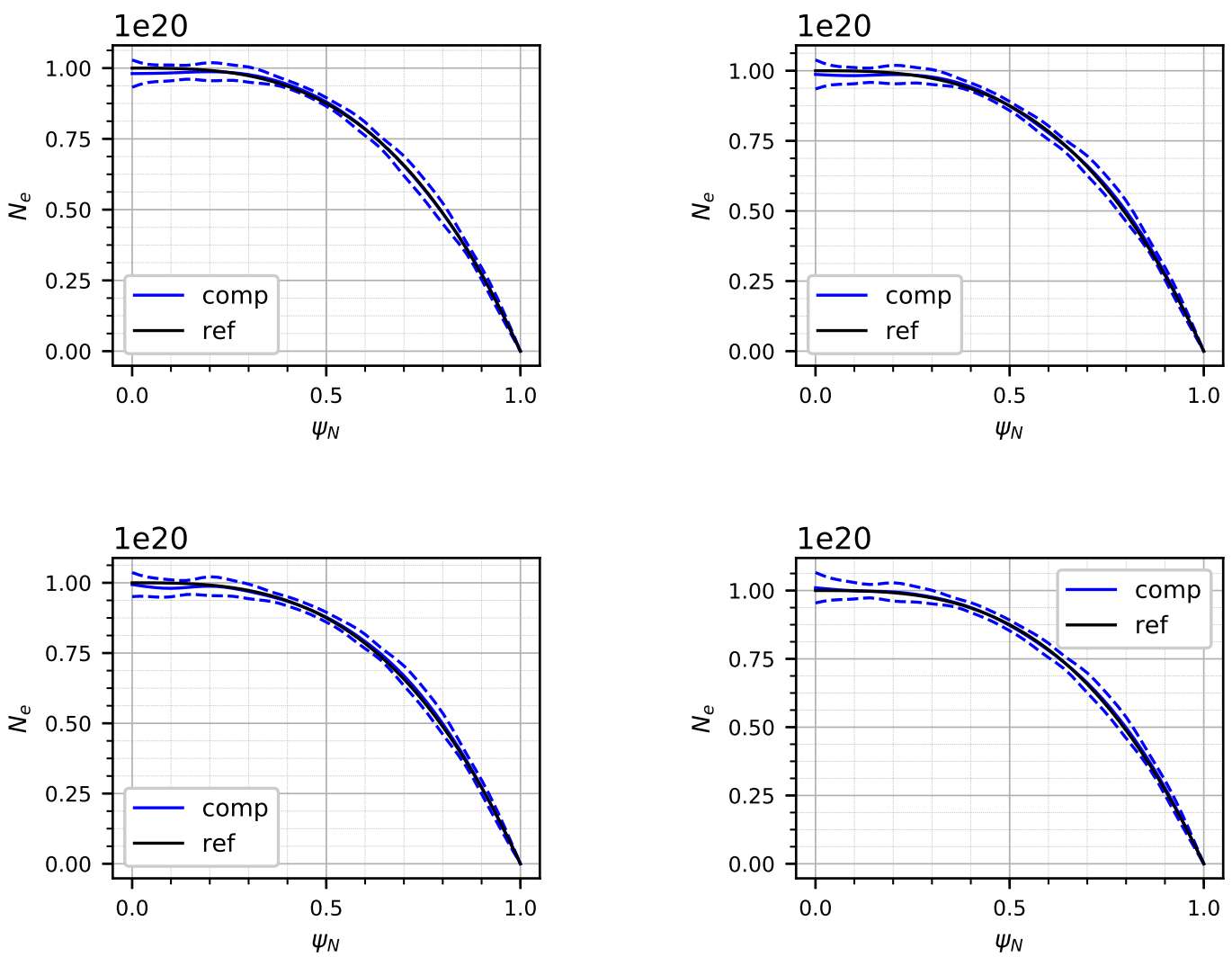

Figure 8. Mean (over 10 reconstructions) identified $N_{e}$ profile and error bar profiles, reference $N_{e}^{r e f}$ profile for $\lambda_{T_{e}}=5,10,20$ and 30 (top left to bottom right). Experiments with $10 \%$ additional noise on interferometry and polarimetry measurements. 

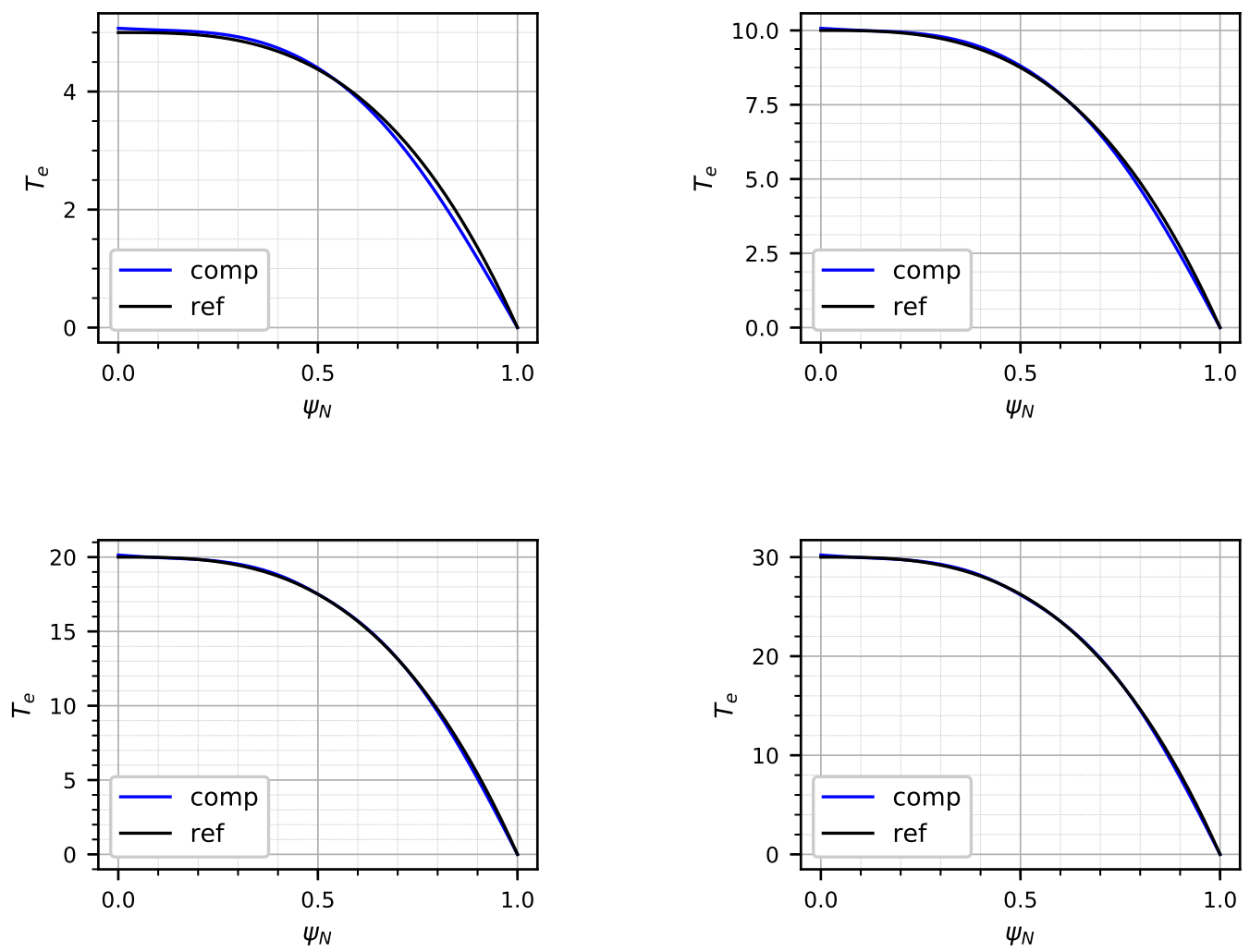

Figure 9. Identified and reference $T_{e}$ profile for $\lambda_{T_{e}}=5,10,20$ and 30. Experiments without additional noise on interferometry and polarimetry measurements. 

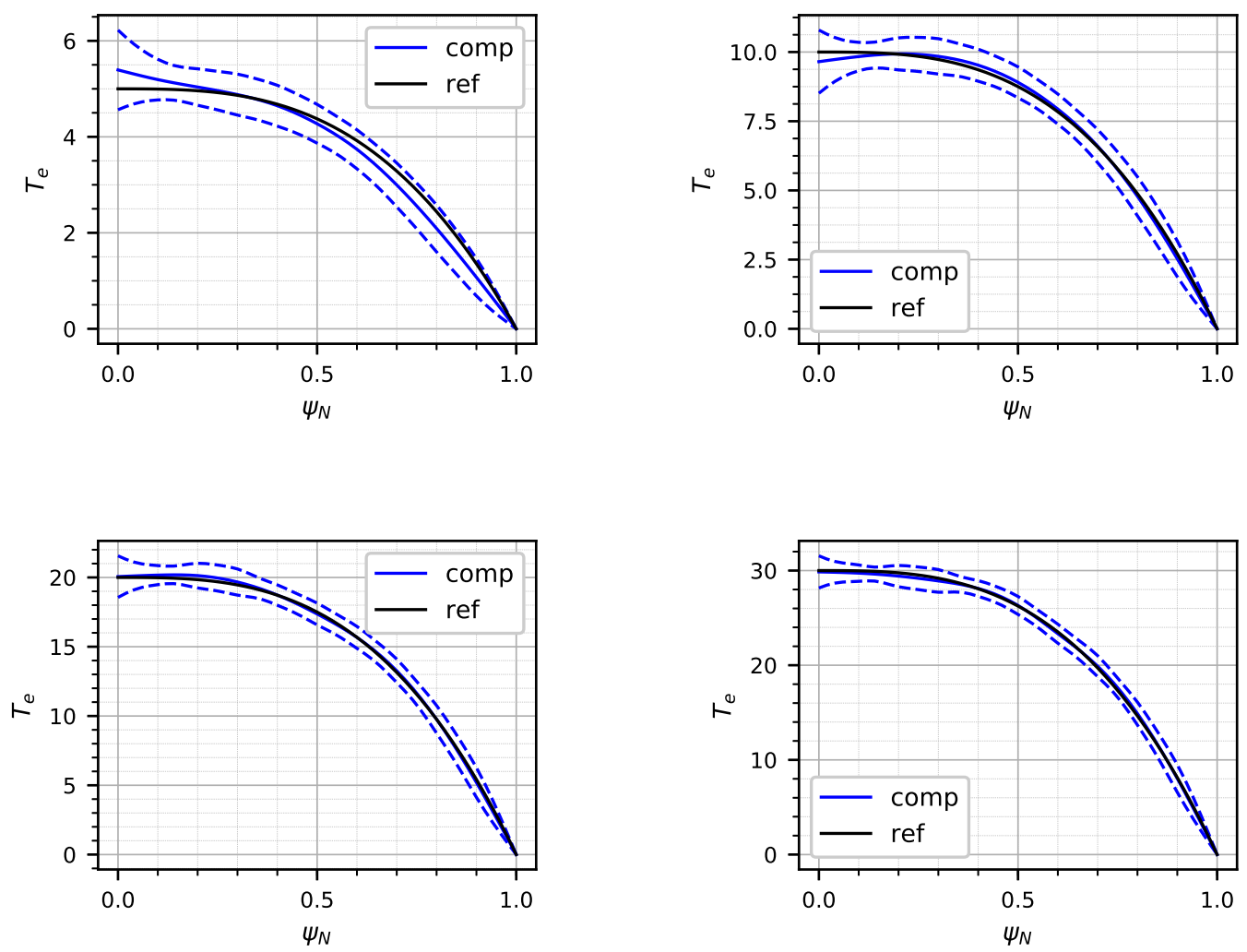

Figure 10. Mean (over 10 reconstructions) identified $T_{e}$ profile and error bar profiles, reference $T_{e}^{r e f}$ profile for $\lambda_{T_{e}}=5,10,20$ and 30. Experiments with $1 \%$ additional noise on interferometry and polarimetry measurements. 

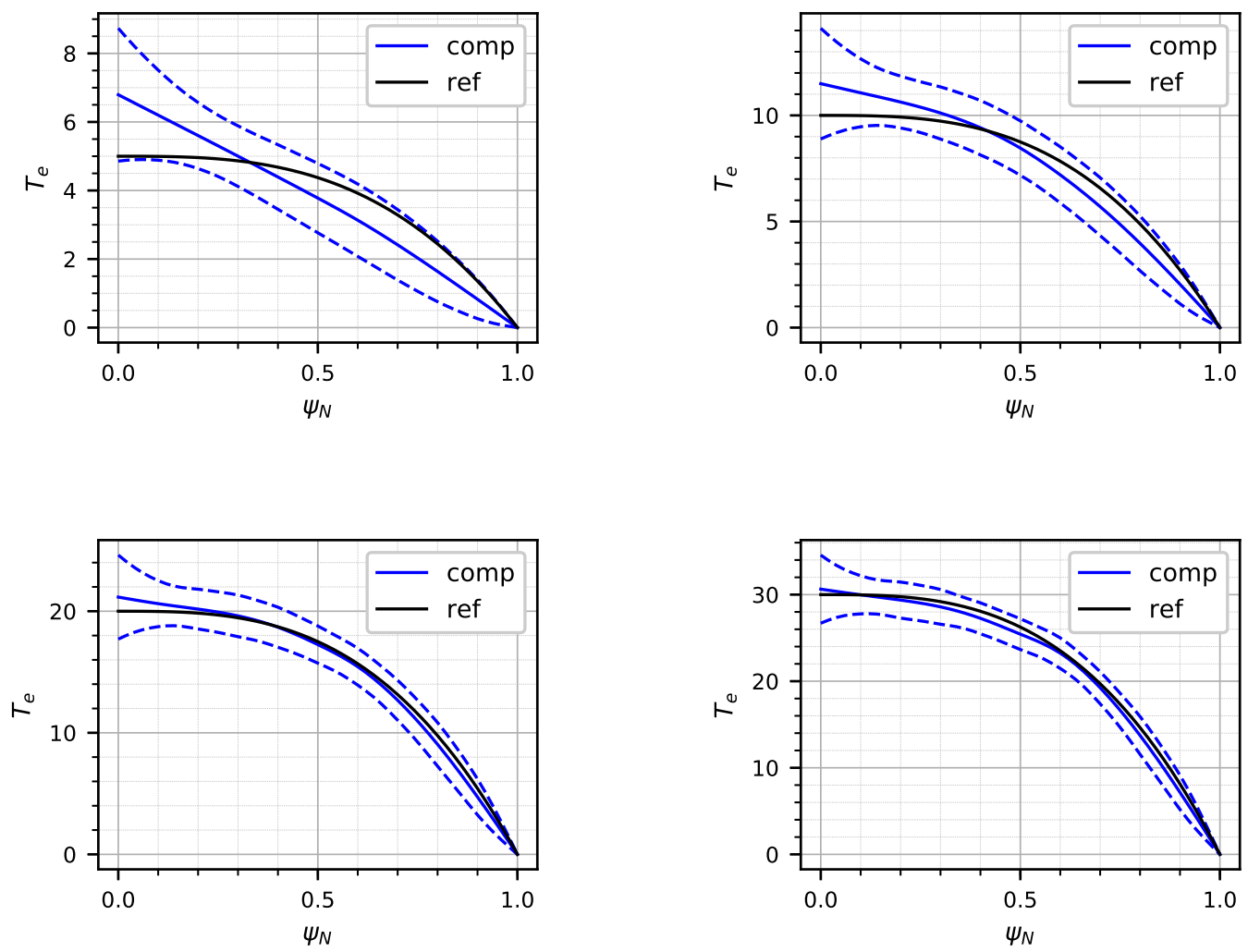

Figure 11. Mean (over 10 reconstructions) identified $T_{e}$ profile and error bar profiles, reference $T_{e}^{r e f}$ profile for $\lambda_{T_{e}}=5,10,20$ and 30. Experiments with 5\% additional noise on interferometry and polarimetry measurements. 

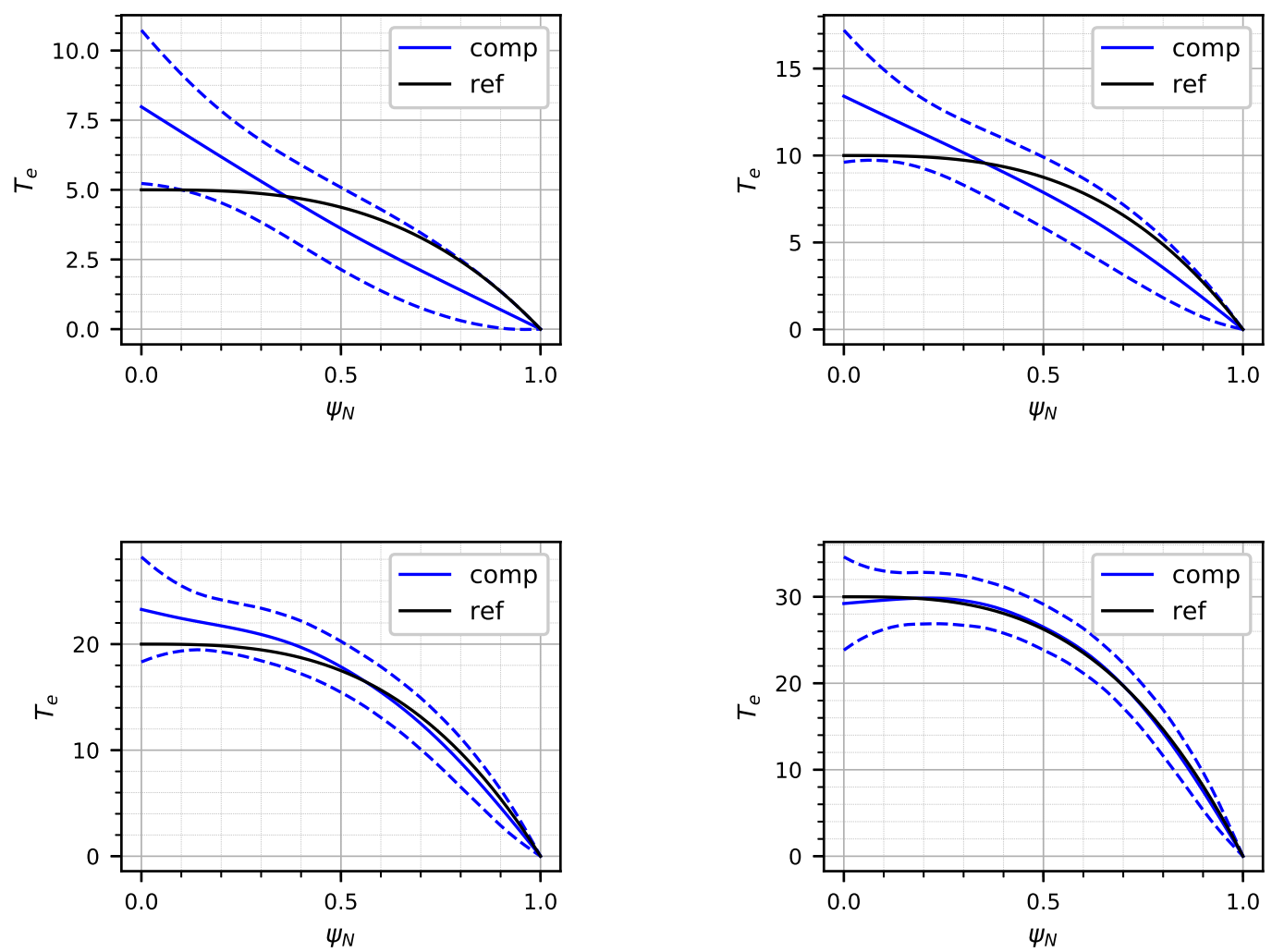

Figure 12. Mean (over 10 reconstructions) identified $T_{e}$ profile and error bar profiles, reference $T_{e}^{r e f}$ profile for $\lambda_{T_{e}}=5,10,20$ and 30. Experiments with $10 \%$ additional noise on interferometry and polarimetry measurements. 


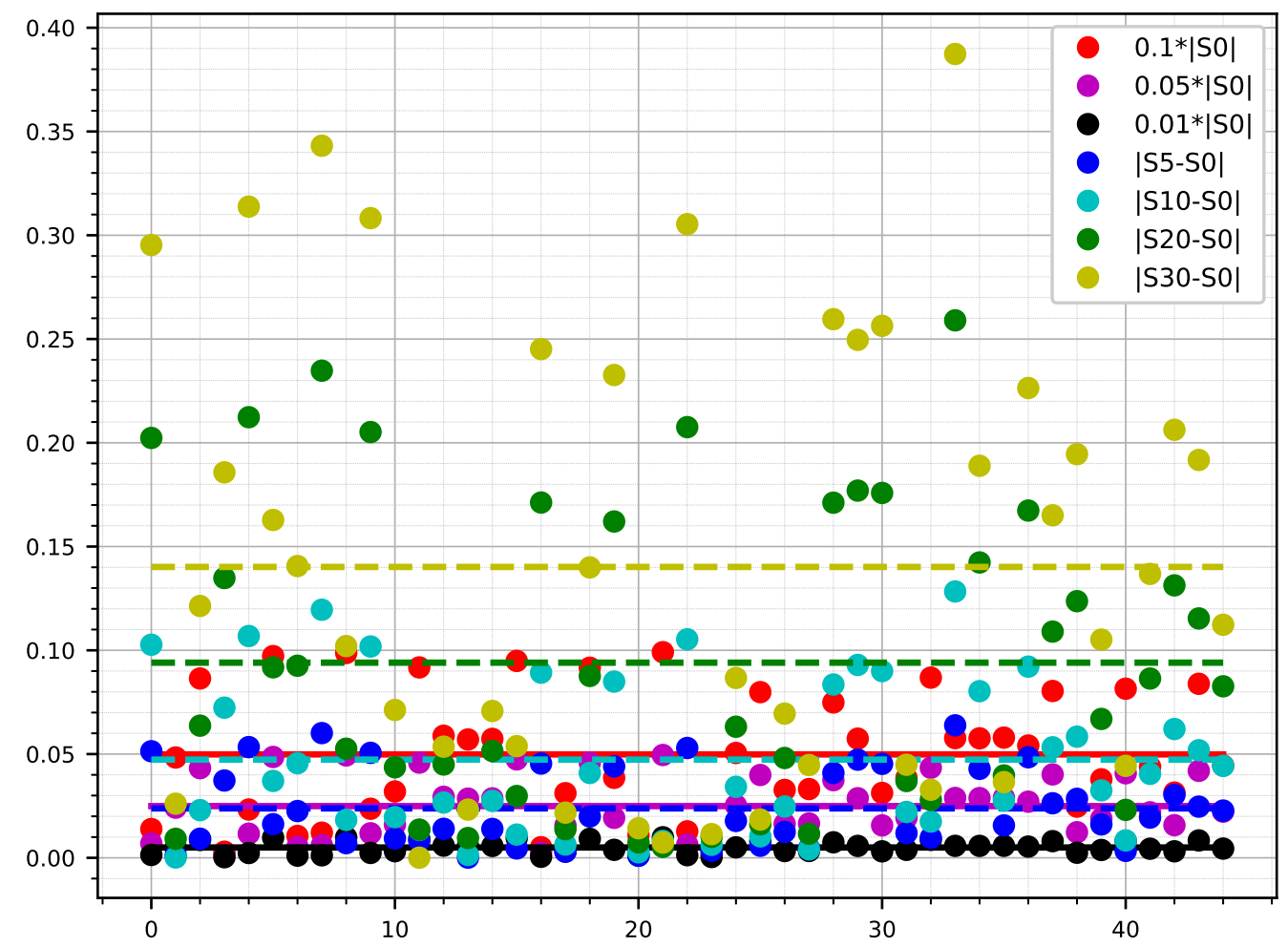

Figure 13. For each of the 3 components of the Stokes vector for each of the 15 chords (45 values on the $\mathrm{X}$-axis) are plotted: 0.1 and 0.01 times the result of the model without $T_{e}$ (denoted $|S 0|$ ) as well as the difference with the model with $T_{e}$ for $\lambda_{T_{e}}=5,10,20$ and 30 . The horizontal line shows the mean value over the 45 components. The mean values $|S 5-S 0|$ and $|S 10-S 0|$ are lower than $0.1 \times|S 0|$. This shows that $10 \%$ noise on Stokes vector measurements can hide the influence of $T_{e}$ on the model. 


\section{Conclusion}

In this study we have presented the first attempt to reconstruct a free-boundary plasma equilibrium and to identify the $T_{e}$ profiles using noisy magnetics, interferometry and polarimetry Stokes vector measurements. The method has been implemented in the code NICE. The interfero-polarimetry formulation explicitly takes into account dependence on electron temperature. This is relevant for foreseen ITER plasma scenarios. Numerical experiments show that for an ITER plasma the identification of the $T_{e}$ profile from noisy measurements is possible. Its quality increases with $T_{e}$ values thus with the importance of the $T_{e}$ perturbation on interfero-polarimetry measurements. 
[1] Sergio E Segre. A review of plasma polarimetry - theory and methods. Plasma Phys. Control Fusion, 41(2):R57, 1999.

[2] V.V Mirnov, W.X. Ding, D.L. Brower, M.A. Van Zeeland, and T.N. Carlstrom. Finite electron temperature effects on interferometric and polarimetric measurements in fusion plasmas. Phys. Plasmas, 14:102105, 2007.

[3] F.P. Orsitto, A. Boboc, C. Mazzotta, E. Giovannozzi, L. Zabeo, and JET EFDA Contributors. Modelling of polarimetry measurements at JET. Plasma Phys. Control Fusion, 50:115009, 2008.

[4] F.P. Orsitto, A. Boboc, P. Gaudio, M. Gelfusa, E. Giovannozzi, C. Mazzotta, A. Murari, and JET EFDA Contributors. Mutual interaction of faraday rotation and cotton-mouton phase shift in JET polarimetric measurements. Rev. Sci. Instrum., 81:10D533, 2010.

[5] F.P. Orsitto, A. Boboc, P. Gaudio, M. Gelfusa, E. Giovannozzi, C. Mazzotta, A. Murari, and JET EFDA Contributors. Analysis of faraday rotation in JET polarimetry measurements. Plasma Phys. Control Fusion, 53:035001, 2011.

[6] R. Imazawa, Y. Kawano, and Y. Kusama. A new approach of equilibrium reconstruction for ITER. Nuclear Fusion, 51:113022, 2011.

[7] R. Imazawa, Y. Kawano, and Y. Kusama. Separation of finite electron temperature effect on plasma polarimetry. Rev. Sci. Instrum., 83:123507, 2012.

[8] J. Chrzanowski and Y.A. Kravtsov. A new method of determining the parameters of thermonuclear plasma on the basis of multichannel polarimetric measurements. Nukleonika, 58(2):281-285, 2013.

[9] B. Faugeras, J. Blum, H. Heumann, and C. Boulbe. Optimal control of a coupled partial and ordinary differential equations system for the assimilation of polarimetry stokes vector measurements in tokamak free-boundary equilibrium reconstruction with application to ITER. Comput. Phys. Comm., 217(Supplement C):43 - 57, 2017.

[10] B. Faugeras, F. Orsitto, and JET Contributors. Equilibrium reconstruction at JET using Stokes model for polarimetry. Nuclear Fusion, 58(10):106032, 2018.

[11] B. Faugeras, J. Blum, C. Boulbe, P. Moreau, and E. Nardon. 2D interpolation and extrapolation of discrete magnetic measurements with toroidal harmonics for equilibrium reconstruction in a Tokamak. Plasma Phys. Control Fusion, 56:114010, 2014.

[12] J. Blum, J. Le Foll, and B. Thooris. The self-consistent equilibrium and diffusion code SCED. Comput. Phys. Comm., 24:235 - 254, 1981.

[13] R. Albanese, J. Blum, and O. Barbieri. On the solution of the magnetic flux equation in an infinite domain. In EPS. 8th Europhysics Conference on Computing in Plasma Physics (1986), pages 41-44, 1986.

[14] H. Heumann, J. Blum, C. Boulbe, B. Faugeras, G. Selig, J.-M. Ané, S. Brémond, V. Grangirard, P. Hertout, and E. Nardon. Quasi-static free-boundary equilibrium of toroidal plasma with CEDRES++: computational methods and applications. J. Plasma Physics, 81(3):905810301, 2015.

[15] J. Nocedal and S. J. Wright. Numerical optimization. Springer Series in Operations Research and Financial Engineering. Springer, New York, second edition, 2006.

[16] M. Hinze, R. Pinnau, M. Ulbrich, and S. Ulbrich. Optimization with PDE constraints, volume 23 of Mathematical Modelling: Theory and Applications. Springer, New York, 2009. 